\title{
Surgical treatment for neuroendocrine liver metastasis: moving ahead in controversy
}

\author{
Jun-Xi Xiang, Yi Lv, Xu-Feng Zhang \\ Department of Hepatobiliary Surgery and Institute of Advanced Surgical Technology and Engineering, The First Affiliated Hospital of Xi'an \\ Jiaotong University, Xi'an, China \\ Correspondence to: Xu-Feng Zhang, MD, PhD. Professor, Department of Hepatobiliary Surgery, The First Affiliated Hospital of Xi'an Jiaotong \\ University, 277 West Yanta Road, Xi'an, China. Email: xfzhang125@xjtu.edu.cn. \\ Comment on: Cloyd JM, Ejaz A, Konda B, et al. Neuroendocrine liver metastases: a contemporary review of treatment strategies. Hepatobiliary Surg \\ Nutr 2020;9:440-51.
}

Submitted Sep 02, 2021. Accepted for publication Oct 18, 2021.

doi: $10.21037 / \mathrm{hbsn}-21-360$

View this article at: https://dx.doi.org/10.21037/hbsn-21-360

Despite the indolent nature of neuroendocrine tumors (NETs), $45-90 \%$ of patients experience neuroendocrine liver metastasis (NELM) during the disease course (1). These patients will develop systemic symptoms secondary to the liver lesions, decreasing both quality of life and prognosis (2). Frilling et al. reported a 5-year overall survival (OS) of $13-54 \%$ in NELM patients, which was significantly lower than the $75-99 \%$ OS for patients without hepatic metastases (3). Surgical treatment plays an important role and has been accepted as the only potential curative option for appropriately selected patients with NELM. However, controversy continues to exist regarding indications for surgery and effectiveness. Over the past few years, a variety of treatment options have improved the outcomes of NELM patients, including surgery, non-operative hepatodirected treatment, and systemic therapy. As Cloyd et al. stated in a current review of NELM management (4), a multidisciplinary and comprehensive evaluation is important when selecting patients for appropriate treatment approaches. Herein, we summarize some key issues related to surgical treatment for NELM that need to be resolved in future research.

Several studies have reported therapeutic benefits of surgery for NELM, and surgical resection is widely employed when feasible. An international multicenter review of over 300 NELM patients undergoing hepatectomy reported a 5 -year survival of $74 \%$, which was superior to the OS of $30 \%$ in patients treated with intra-arterial therapies (1). In contrast, a systematic review found no evidence for long-term survival benefit of liver resection compared with any other liver-directed therapies (5). Since prospective randomized controlled trials are unlikely to be conducted, the dispute will continue. The fundamental reason behind the controversy is the range of surgical indications. The impact of hepatectomy on long-term prognosis is still difficult to assess due to potential selection bias for different treatments. For example, patients with heavier disease burden, worse performance status, and severe comorbidities tend to receive conservative treatment and are more likely to have poor outcomes. The European Neuroendocrine Tumor Society (ENETS) guidelines recommend hepatic resection for NELM patients as long as R0 resection is feasible, tumors are classified as grade 1 or 2 , there are no extrahepatic metastases, and perioperative morbidity is $<30 \%$ and mortality $<5 \%$ (5). However, due to the heterogeneity of NETs, "off-label" indications for hepatectomy are not uncommon.

Debulking is gradually being an accepted treatment strategy for patients who cannot undergo complete resection of all lesions. Previous studies have reported comparable OS in patients receiving cytoreductive surgery in which $70-90 \%$ of lesions were resected (6). Specifically, there is ongoing debate over resection of the primary tumor in the setting of unresectable metastasis. While improvement of long-term survival was observed in a systematic review of primary tumor resection for unresectable NELM, other research has reported limited benefit for symptomatic patients (7). The proposed rationale behind the approach is to relieve symptoms caused by the primary tumor, prevent 
the future possibility of obstruction and bleeding, obtain histological diagnosis, and potentially improve outcomes. However, there are no randomized controlled trials and only 1 prospective observational study currently registered on Clinicaltrials.gov (NCT03442959) to identify the specific issue of survival benefits of primary resection in the case of unresectable metastasis, and therefore the findings need to be interpreted cautiously. Taken together, rather than routine primary resection for patients with asymptomatic NELM, it would be more appropriate to conduct a multidisciplinary discussion involving careful consideration of the patient's physical status and comorbidities.

There is also controversy over whether hepatectomy should be performed in NELM patients with unresectable primary tumor and in the case of extrahepatic metastasis. Our previous study revealed similar long-term outcomes in NELM patients with primary tumor resection versus those without resection (8). In contrast, compared to patients with intrahepatic-only disease, patients with extrahepatic metastases had a risk of death that was 2.5 times higher (8). However, a median OS of 87 months was observed in patients with extrahepatic disease who received liver-directed therapies for NELM (9). In addition, the survival of patients with extrahepatic metastasis undergoing surgical resection was significantly higher than that of patients receiving other treatments (10). Given that the majority of NELM patients eventually die from hepatic dysfunction, aggressive treatment may be required, even in cases of extrahepatic lesions or unresectable primary disease. Further studies are needed to investigate the benefits of surgical resection and minimally invasive treatment such as intra-arterial therapy and concomitant ablation for patients with a heavy burden of disease.

Although NETs are often considered a low-grade malignancy, some NELM patients will relapse, and thus aggressive resection cannot be considered long-term curative. Recurrent disease prediction and management are key issues for NELM patients after initial resection. Some studies have found recurrence rates of $70-95 \%$ in patients undergoing initial resection for NELM (4). Among patients who experienced recurrence, almost $40 \%$ relapsed within 1 year and $40 \%$ within 3 years (4). Collectively, postoperative recurrence was significantly associated with morphological (tumor size, number, lymph metastasis, extrahepatic disease) and biological (differentiation, ki-67 index, synchronous disease, nonfunctional status, primary location) features of disease and treatment modality (major hepatectomy, margin, adjuvant therapy) (10). After recurrence, repeat resection is safe and feasible and may achieve good longterm prognosis in carefully selected patients $(2,11)$. Nonsurgical treatments such as octreotide, chemotherapy, molecular-targeting therapy, and liver-directed treatments are alternative options, and these have been investigated in different cohorts (11). Therefore, the treatment strategy for recurrent NELM should be individually tailored and involve discussion among oncologists, gastroenterologists, and surgeons.

Liver transplantation is a definitive treatment for unresectable NELM in the setting of resected primary NET and absence of extrahepatic disease (4). Under strict selection criteria, one previous study presented 5 -year OS and disease-free survival (DFS) rates of $97 \%$ and $89 \%$, respectively, in patients undergoing liver transplantation for recurrent NELM (12). Similarly, the European Liver Transplant Registry study presented a 5 -year OS of $59 \%$ post-transplantation for recurrent NELM (13). When researchers applied the Milan criteria retrospectively, the calculated OS improved to $79 \%$ but came at the expense of excluding $64 \%$ of patients. The need for such a rigorous selection process is debatable and expansion of the Milan criteria was recently suggested. In addition to standardization of patient selection, future studies on multidisciplinary approaches, posttransplant immunosuppressants, and perioperative medical therapies for NELM are needed.

Over the last few years, the rapid development of systemic therapies has significantly improved the longterm survival and quality of life for NELM patients. Adjuvant and neoadjuvant therapies highlighted possible changes of surgical indications, timing, and management of advanced and/or recurrent disease. In addition to chemotherapy, current options for systemic therapy include local interventional treatment [conventional transarterial chemoembolization (TACE), transarterial embolization (TAE), transarterial radioembolization (TARE), radiofrequency ablation (RFA), and microwave ablation (MWA)], inhibition of mammalian target of rapamycin (mTOR) and tyrosine kinases to limit tumor growth and malignant transformation, somatostatin analogs (SSA) that can reduce functional output and have an antiproliferative effect, and immunotherapy that regulates the response of the immune system to antineoplastic drugs (14). Despite the variety of armamentarium available, the optimal treatment regimen remains uncertain. An obvious question involves the combination, timing, and sequence of treatments available. Moreover, effective biomarkers are needed to aid 
in individualizing treatment decisions in NELM. Thus, the appropriate selection and sequencing of treatment approaches relies mostly on clinical judgment while investigations of combined therapy are still underway (15).

Although abundant knowledge of NELM is currently available, many questions and debates remain regarding surgical treatment for NELM, especially for advanced tumors. The optimal therapy should be personalized and consider a variety of factors, including biological tumor features, liver metastasis patterns, extrahepatic disease, patient characteristics, as well as outcome perspective.

\section{Acknowledgments}

Funding: None.

\section{Footnote}

Provenance and Peer Review: This article was commissioned by the editorial office, Hepatobiliary Surgery and Nutrition. The article did not undergo external peer review.

Conflicts of Interest: All authors have completed the ICMJE uniform disclosure form (available at https://hbsn. amegroups.com/article/view/10.21037/hbsn-21-360/coif). The authors have no conflicts of interest to declare.

Ethical Statement: The authors are accountable for all aspects of the work in ensuring that questions related to the accuracy or integrity of any part of the work are appropriately investigated and resolved.

Open Access Statement: This is an Open Access article distributed in accordance with the Creative Commons Attribution-NonCommercial-NoDerivs 4.0 International License (CC BY-NC-ND 4.0), which permits the noncommercial replication and distribution of the article with the strict proviso that no changes or edits are made and the original work is properly cited (including links to both the formal publication through the relevant DOI and the license). See: https://creativecommons.org/licenses/by-nc-nd/4.0/.

\section{References}

1. Mayo SC, Pawlik TM. Surgical management of neuroendocrine tumors: treatment of localized and metastatic disease. Oncology (Williston Park) 2011;25:806, 808,810 .
2. Frilling A, Clift AK. Therapeutic strategies for neuroendocrine liver metastases. Cancer 2015;121:1172-86.

3. Frilling A, Modlin IM, Kidd M, et al. Recommendations for management of patients with neuroendocrine liver metastases. Lancet Oncol 2014;15:e8-21.

4. Cloyd JM, Ejaz A, Konda B, et al. Neuroendocrine liver metastases: a contemporary review of treatment strategies. Hepatobiliary Surg Nutr 2020;9:440-51.

5. Lesurtel M, Nagorney DM, Mazzaferro V, et al. When should a liver resection be performed in patients with liver metastases from neuroendocrine tumours? A systematic review with practice recommendations. HPB (Oxford) 2015;17:17-22.

6. Morgan RE, Pommier SJ, Pommier RF. Expanded criteria for debulking of liver metastasis also apply to pancreatic neuroendocrine tumors. Surgery 2018;163:218-25.

7. Tsilimigras DI, Ntanasis-Stathopoulos I, Kostakis ID, et al. Is Resection of Primary Midgut Neuroendocrine Tumors in Patients with Unresectable Metastatic Liver Disease Justified? A Systematic Review and Meta-Analysis. J Gastrointest Surg 2019;23:1044-54.

8. Xiang JX, Zhang XF, Beal EW, et al. Hepatic Resection for Non-functional Neuroendocrine Liver Metastasis: Does the Presence of Unresected Primary Tumor or Extrahepatic Metastatic Disease Matter? Ann Surg Oncol 2018;25:3928-35.

9. Saxena A, Chua TC, Perera $M$, et al. Surgical resection of hepatic metastases from neuroendocrine neoplasms: a systematic review. Surg Oncol 2012;21:e131-41.

10. Spolverato G, Bagante F, Aldrighetti L, et al. Neuroendocrine Liver Metastasis: Prognostic Implications of Primary Tumor Site on Patients Undergoing Curative Intent Liver Surgery. J Gastrointest Surg 2017;21:2039-47.

11. Spolverato G, Bagante F, Aldrighetti L, et al. Management and outcomes of patients with recurrent neuroendocrine liver metastasis after curative surgery: An international multi-institutional analysis. J Surg Oncol 2017;116:298-306.

12. Mazzaferro V, Sposito C, Coppa J, et al. The Long-Term Benefit of Liver Transplantation for Hepatic Metastases From Neuroendocrine Tumors. Am J Transplant 2016;16:2892-902.

13. Olausson M, Friman S, Herlenius G, Cahlin C, Nilsson $\mathrm{O}$, Jansson S, Wängberg B, Ahlman H. Orthotopic liver or multivisceral transplantation as treatment of metastatic neuroendocrine tumors. Liver Transpl 2007;13:327-33. 
14. Scoville SD, Cloyd JM, Pawlik TM. New and emerging systemic therapy options for well-differentiated gastroenteropancreatic neuroendocrine tumors. Expert Opin Pharmacother 2020;21:183-91.

Cite this article as: Xiang JX, Lv Y, Zhang XF. Surgical treatment for neuroendocrine liver metastasis: moving ahead in controversy. HepatoBiliary Surg Nutr 2021;10(6):868-871. doi: 10.21037/hbsn-21-360
15. Rodriguez-Freixinos V, Thawer A, Capdevila J, et al. Advanced Pancreatic Neuroendocrine Neoplasms: Which Systemic Treatment Should I Start With? Curr Oncol Rep 2021;23:80. 